\title{
Identification of RAD54 homolog B as a promising therapeutic target for breast cancer
}

\author{
JING FENG ${ }^{1,2^{*}}$, JUANJUAN HU ${ }^{1,2 *}$ and YING XIA ${ }^{1,2}$ \\ ${ }^{1}$ Institute of Chemical Component Analysis of Traditional Chinese Medicine, Chongqing Medical and \\ Pharmaceutical College; ${ }^{2}$ Engineering Research Center of Pharmaceutical Sciences, Chongqing 401331, P.R. China
}

Received January 24, 2019; Accepted July 26, 2019

DOI: $10.3892 / \mathrm{ol} .2019 .10854$

\begin{abstract}
Breast cancer is a recognized threat to the health of women globally. Due to the lack of the knowledge about the molecular pathogenesis of breast cancer, therapeutic strategies remain inadequate, especially for aggressive breast cancer. In the present study, sequential bioinformatics analysis was performed using data from the GSE20711 dataset, and the results demonstrated that three genes may impact the survival of patients with breast cancer. One of these genes, RAD54 homolog B (RAD54B), may be a potential prognostic factor for breast cancer. A signature was established that could evaluate the overall survival for patients with breast cancer based on the risk score calculated from RAD54B expression and the Tumor-Node-Metastasis (TNM) stage [risk score $=\operatorname{expRAD54B} \times 0.236+\mathrm{TNM}$ stage $(\mathrm{I} / \mathrm{II}=0$ or III/IV=1) x1.025]. In addition, based on the GSE85871 dataset and inhibitory assay, the study identified a natural compound, Japonicone A, which may reduce the proliferation of breast cancer cells by inhibiting the expression of RAD54B. Overall, the present study identified a novel candidate gene and a candidate compound as promising therapeutic targets for the treatment of breast cancer.
\end{abstract}

Correspondence to: Professor Ying Xia, Institute of Chemical Component Analysis of Traditional Chinese Medicine, Chongqing Medical and Pharmaceutical College, 82 Daxuecheng Middle Road, Shapingba, Chongqing 401331, P.R. China

E-mail: 370386006@qq.com

${ }^{*}$ Contributed equally

Abbreviations: WGCNA, weighted gene co-expression network analysis; GO, Gene Ontology; KEGG, Kyoto Encyclopedia of Genes and Genomes; BP, biological process; TCGA, The Cancer Genome Atlas; MF, molecular function; CC, cellular component; DEGs, differentially expressed genes

Key words: breast cancer, RAD54 homolog B, proliferation, Japonicone A

\section{Introduction}

Cancer is considered to be one of the most dangerous factors to human life. The global cancer statistics for 2018 demonstrated that breast cancer exhibits the highest morbidity and mortality rates in females worldwide compared with other types of cancer (1). Several therapeutic strategies have been developed for breast cancer treatment, including surgery, chemotherapy, radiotherapy, hormone therapy and newly improved immunotherapy (2). However, due to the high heterogeneity among different types of breast cancer, the prognosis for a number of patients is still poor, especially for patients with distant metastases, who are usually diagnosed at a late stage (3). As a result, it is very important to identify the basic molecular mechanisms leading to the occurrence and development of breast cancer. Improved knowledge on breast cancer may result in more efficient treatment strategies.

With the development of new research techniques, bioinformatics analysis is considered as one of the most important methods used to study cancer, especially the underlying molecular mechanisms (4). A number of studies have used bioinformatics to analyze the gene expression profiles of various types of cancer by identifying, comparing or clustering differentially expressed genes in cancer cells compared with healthy cells. Candidate genes involved in the occurrence and development of cancer can be identified and further studied to be developed as promising therapeutic targets (5-8). Bioinformatics analysis is considered to be an important technique in breast cancer study and has already helped achieve promising improvements, such as identifying new prognostic factors or pathways and genes associated with breast cancer (9-12).

The present study analyzed the expression data from GSE20711 and revealed that the RAD54B gene was associated with the Tumor-Node-Metastasis (TNM) stage, which may be used as a signature for predicting the overall survival time of patients with breast cancer. This signature may contribute to the precise treatment and prognostic monitoring of patients with breast cancer. Furthermore, the present study identified a compound (Japonicone A) from the traditional herb Inula japonica Thunb that could decrease the proliferation of breast cancer cells by inhibiting the expression of RAD54B. The present study identified a novel candidate gene and a 
candidate compound as promising therapeutic targets for the treatment of breast cancer.

\section{Materials and methods}

Gene expression datasets. The gene expression datasets GSE20711 and GSE85871 were downloaded from the Gene Expression Omnibus database (https://www.ncbi.nlm.nih. gov/geo). GSE20711 was comprised of 88 breast cancer samples and 2 normal breast tissue samples, and used the platform GPL570 (Affymetrix Human Genome U133 Plus 2.0 Array) (13). GSE85871 was comprised of the gene expression profiles of MCF-7 cells, which were treated with 102 different molecules used in traditional Chinese medicine, and used the GPL571 platform (Affymetrix Human Genome U133A 2.0 Array) (14). The Cancer Genome Atlas (TCGA) Breast Invasive Carcinoma dataset (including high-throughput sequencing (HTSeq) and clinical data of 1,104 breast cancer tissue samples and 113 normal breast samples) was downloaded using the $\mathrm{R}$ package 'TCGAbiolinks (version 2.10.0)' (15).

Screening for differentially expressed genes (DEGs). The 'limma (version 3.36.2)' package was used to load normalized data into R (version 3.3.3; https://www.r-project.org) software and screen the DEGs between breast cancer and non-tumor tissues (16). The genes with fold-change $\geq 2$ and an adjusted $\mathrm{P}$-value (false discovery rate) $<0.05$ were identified as DEGs (17).

Co-expression network construction and module identification. Weighted correlation network analysis (WGCNA) is a commonly used systemic biological data mining method for describing the correlation patterns among genes and identifying the modules of highly correlated genes; it uses average linkage hierarchical clustering coupled with topological overlap dissimilarity based on high-throughput chip or RNA-Seq data (18). The 'WGCNA (version 1.63)' package in R was used to construct the co-expression network for the DEGs in the 88 breast cancer samples in GSE20711 (18). $\beta$ is a soft-thresholding parameter that emphasizes strong correlations between genes and depreciates weak correlations (19). In the present study, $\beta=18$ (scale-free $\mathrm{R}^{2}=0.8$ ) was used to ensure a scale-free network. A cut height of 0.85 and effect size of $\geq 10$ were used to identify the modules. Pearson's correlation matrices were calculated for the modules (20).

Enrichment analysis. The Gene Ontology (GO) and Kyoto Encyclopedia of Genes and Genomes (KEGG) pathway enrichment analysis of the genes in the modules were performed using the 'clusterProfiler (version 3.9.1)' $\mathrm{R}$ package based on hypergeometric distribution algorithm $(\mathrm{P}<0.05)$, and 'GOplot (version 1.0.2)' was used for further analysis $(21,22)$.

Survival analysis. To validate the genes in the turquoise modules, the largest modules, the clinical information and RNA sequencing data (HTSeq-FPKM) of breast cancer were obtained from TCGA Project database (https://cancergenome. nih.gov). Kaplan-Meier survival analysis with the log-rank test was conducted to evaluate the association between the genes in the turquoise module and patient survival. $\mathrm{P}<0.05$ was considered to indicate a statistically significant association. Univariate Cox analysis was used to test whether the genes may be used as independent prognostic factors. The data were randomly divided into two groups: A discovery cohort $(n=458)$ and an internal testing cohort $(n=457)$. The genes and clinicopathological characteristics that were significant in the univariate Cox analysis and the Kaplan-Meier survival analysis were used for the multivariate Cox regression analysis. The Akaike information criterion (AIC) value, which was calculated based on different influencing factors by the multivariate Cox regression analysis, was used to remove the confounding factors to obtain the best variable for data fitting, where the minimum AIC value has the best fit (23). Subsequently, a prognostic mRNA and clinical trait signature with min AUC value was constructed, which may be used to calculate a risk score for each individual patient with breast cancer. According to the median of the risk score (median value, 1.915), the patients were stratified into low-risk and high-risk groups. The risk groups from the two cohorts were evaluated using Kaplan-Meier analysis and the log-rank test (24). A nomogram combining the risk score with two other clinical factors (age and sex) was constructed to provide a graphic representation of the prediction model using the $\mathrm{R}$ package rms (version 5.1-3.1) (25).

Protein-protein interaction (PPI) network construction. The search tool for the retrieval of interacting genes/proteins (STRING) database (https://string-db.org/) was used to construct the PPI networks, and the results exported from STRING were imported into Cytoscape (version 3.4.0) for visualization (24).

Immunohistochemistry. The Human Protein Atlas (http://www. proteinatlas.org/) was used to validate the expression of the three genes in breast cancer tissue (26). The direct links to these images are as follows: RAD54B, https://www.proteinatlas.org/ ENSG00000197275-RAD54B/pathology/tissue/breast+cancer \#imid_2186145; KIF21A, https://www.proteinatlas.org/ ENSG00000139116-KIF21A/pathology/tissue/breast+cancer\# imid_17124866; and C8orf76, https://www.proteinatlas.org/ ENSG00000189376-C8orf76/pathology/tissue/breast+cancer\# imid_6235831.

Gene Set Enrichment Analysis (GSEA). GSEA was performed using KEGG pathway annotation data from the KEGG database (27). According to the median value of RAD54B expression, patients from the GSE20711 and TCGA datasets were divided into two groups. The clusterProfiler (version 3.9.1) package was used to analyze the data and construct the ridge plot (28).

Cell culture and stimulation. The MCF-7 cell line was obtained from the American Type Culture Collection. The cells were cultured in complete Dulbecco's modified Eagle's medium (Gibco; Thermo Fisher Scientific, Inc.) supplemented with $10 \%$ fetal bovine serum (Gibco; Thermo Fisher Scientific, Inc.) in $5 \% \mathrm{CO}_{2}$ stored at $37^{\circ} \mathrm{C}$. The cell cultures were checked for Mycoplasma contamination using a Mycoplasma PCR Detection kit (Sigma-Aldrich; Merck KGaA) every 3 months. The cell line was not listed 
in the database of commonly misidentified cell lines (https:// iclac.org/databases/cross-contaminations).

Japonicone A ( $>97 \%$ purity) was kindly gifted by Professor Weidong Zhang (School of Pharmacy, Second Military Medical University, Shanghai, China) (29-32). Japonicone A was dissolved in dimethyl sulfoxide (DMSO) and the solution was diluted in the cell culture media to ensure that the concentration of DMSO was $<0.1 \%$, as described in a previous study (31). The working concentration $(10 \mu \mathrm{M})$ of Japonicone A was the same as the one applied in the GSE85871 dataset, which was initially used to identify that Japonicone A inhibited the expression of RAD5B in MCF-7 cells (15). In addition, $\mathrm{Hu}$ et al (29) used $10 \mu \mathrm{M}$ Japonicone $\mathrm{A}$ in a treatment assay. Preliminary experiments were performed with $10 \mu \mathrm{M}$ Japonicone A on the proliferation of MCF-7 cells in the present study, and the results suggested that the concentration was effective (data not shown). Therefore, MCF-7 cells were treated with $10 \mu \mathrm{M}$ Japonicone A for $24 \mathrm{~h}$ prior to cell collection and analysis. DMSO without Japonicone A was used as the solvent control. The volume of the solvent control was the same with DMSO-dissolved Japonicone A used in the experimental group.

Reverse transcription-quantitative PCR. Total RNA was extracted from MCF-7 cells using TRIzol ${ }^{\circledR}$ reagent (Invitrogen; Thermo Fisher Scientific, Inc.). RNA was reverse-transcribed into cDNA with High-Capacity cDNA Reverse Transcription kit (Applied Biosystems; Thermo Fisher Scientific, Inc.). qPCR was performed using SYBR ${ }^{\circledR}$ Premix Ex Taq II (Takara Bio, Inc.). Relative mRNA expression levels were calculated by normalizing the relative quantitation cycle value to the control group following standardization to the internal control $\beta$-actin (33). The primers used were as follows: Human RAD54B forward, 5'-AAGAACCTGACTGCCTCACG-3' and reverse, 5'-TCCACCACAGGTAAACCAGC-3'; and human $\beta$-actin forward, 5'-CAGGGCGTGATGGTGGGCA-3' and reverse, 5'-CAAACATCATCTGGGTCATCTTCTC-3'. The thermocycling conditions for the RT-qPCR was as follows: $2 \mathrm{~min}$ at $95^{\circ} \mathrm{C}, 40$ cycles at $95^{\circ} \mathrm{C}$ for $10 \mathrm{sec}, 59.5^{\circ} \mathrm{C}$ for $10 \mathrm{sec}$, $68^{\circ} \mathrm{C}$ for $15 \mathrm{sec}$, and $72^{\circ} \mathrm{C}$ for $10 \mathrm{sec}$.

Receiver operating characteristic (ROC) curve analysis. The R package 'survivalROC (version 1.0.3)' was used for the ROC curve analysis (34). The ROC curve was used to test the sensitivity and specificity of the variables in predicting overall survival, and to assess the predictive ability of the calculated prognostic signature for 5-year patient survival (25).

Decision curve analysis (DCA). R package 'rmda (version 1.6)' was used for DCA, which estimates the net benefit of a signature by subtracting the false-positives from the true-positives $(35,36)$.

Flow cytometry. Anti-mouse marker of proliferation Ki-67 (ki-67)-FITC flow cytometry antibody was purchased from Miltenyi Biotec (diluted 1:100 with 1X permeabilization buffer; catalog no. 130-117-691). The intracellular staining of ki-67 was performed using the Foxp3/Transcription Factor Staining Buffer Set (eBioscience; Thermo Fisher Scientific, Inc.) according to the manufacturer's instructions. Briefly,
MCF-7 cells were collected and centrifuged at $150 \mathrm{x} g$ for $5 \mathrm{~min}$ at room temperature. The cell pellets were mixed with $1 \mathrm{ml}$ fixation-permeabilization buffer (a 1:3 mixture of fixation-permeabilization concentrate and diluent, which were included in the kit) at $4^{\circ} \mathrm{C}$ for $1 \mathrm{~h}$. The cells were washed twice with $2 \mathrm{ml} 1 \mathrm{X}$ permeabilization buffer (10X permeabilization buffer diluted to $1 \mathrm{X}$ with $\mathrm{ddH}_{2} \mathrm{O}$ ). The fixed cells were stained with the anti-ki-67-FITC antibody for $30 \mathrm{~min}$ at room temperature in the dark. Finally, the washed cells were resuspended in PBS prior to detection using BD FACSVerse (BD Biosciences). The flow cytometric data were analyzed with FlowJo software (version 10.3; FlowJo LLC).

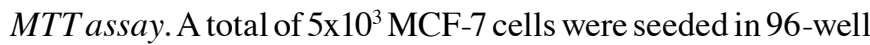
plates and treated with the aforementioned amount of control (DMSO) or $10 \mu \mathrm{M}$ Japonicone A for $24 \mathrm{~h}$. Subsequently, $20 \mu \mathrm{l}$ MTT $(5 \mathrm{mg} / \mathrm{ml})$ was added to the wells and incubated for $4 \mathrm{~h}$. The wells were supplemented with $150 \mu \mathrm{l}$ DMSO to dissolve the formazan crystals prior to optical density measurement by a microplate reader at $490 \mathrm{~nm}$. There were three duplicate wells for each group, and the cell viability was normalized to the control group in each experiment.

Statistical analysis. The statistical analyses were performed using R (version 3.3.3). Student's t-test was used for comparisons between two independent groups. $\mathrm{P}<0.05$ was considered to indicate a statistically significant difference. The experiments presented in Fig. 7 were replicated four times and each dot in the histogram represents one independent experiment; lines and error bars represent the mean \pm SD.

\section{Results}

Co-expression network construction and key module identification. The GSE20711 samples with complete clinical data were included in the WGCNA analysis (Fig. 1A). DEGs between breast cancer tissues and non-tumor tissues were identified (Table SI). Based on the DEGs, a co-expression network was constructed, and the modules were identified by WGCNA. The results revealed that the most significant DEGs could be grouped into three major modules (turquoise, blue and brown modules) (Fig. 1B). Further analysis demonstrated that the turquoise module exhibited the highest positive correlation with TNM stage and negative correlation with ER status compared with the other modules (Fig. 1C). Thus, this module was identified as the clinically significant module for subsequent analysis.

Enrichment analysis and PPI network of the turquoise module. GO enrichment analysis was performed for the genes in the turquoise module, and the results were categorized into three functional groups: Biological process (BP), molecular function (MF) and cellular component (CC). The genes in the BP group were mainly enriched in 'mitotic cell cycle process', 'cell cycle', 'cell cycle process', 'mitotic cell cycle', 'cell division' and 'nuclear division' (Fig. 2A); the genes in the $\mathrm{CC}$ group were enriched in 'condensed chromosome', 'chromosome', 'spindle' and 'chromosomal region' (Fig. 2B); the genes in the MF group were mainly enriched in 'protein binding', 'ATP binding', 'catalytic 
A

\section{Sample dendrogram and trait heatmap}
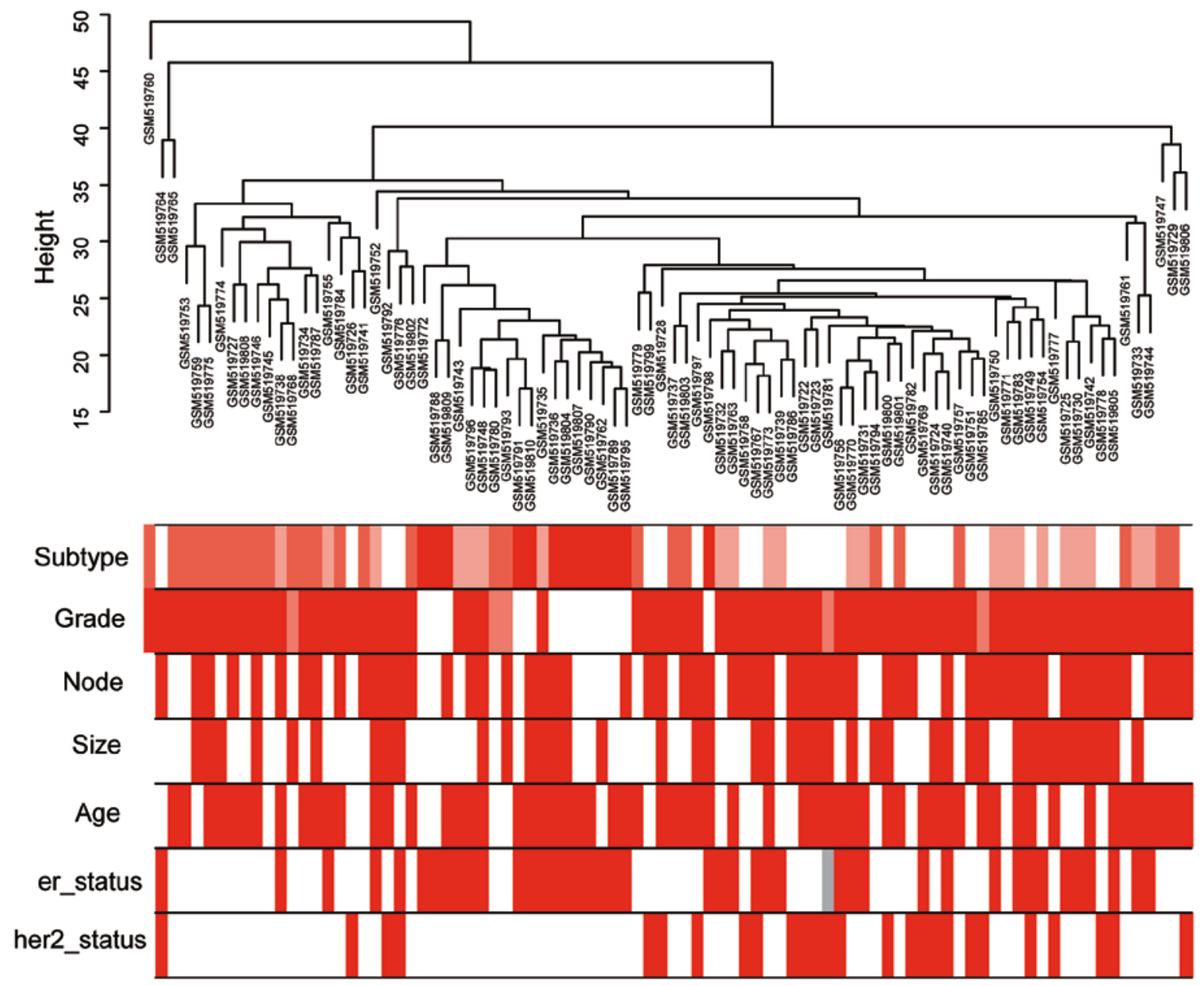

B Cluster Dendrogram

C

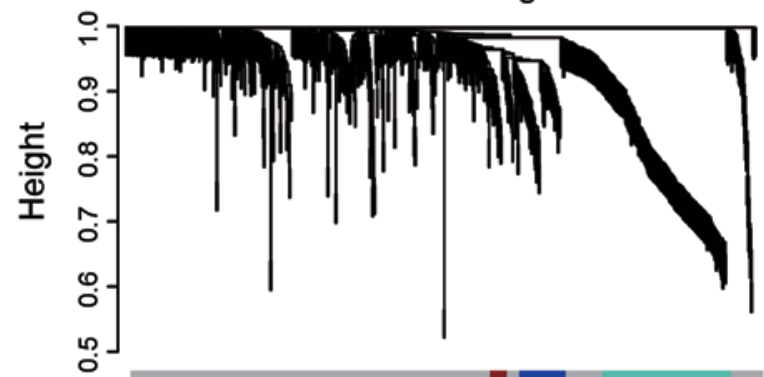

Module

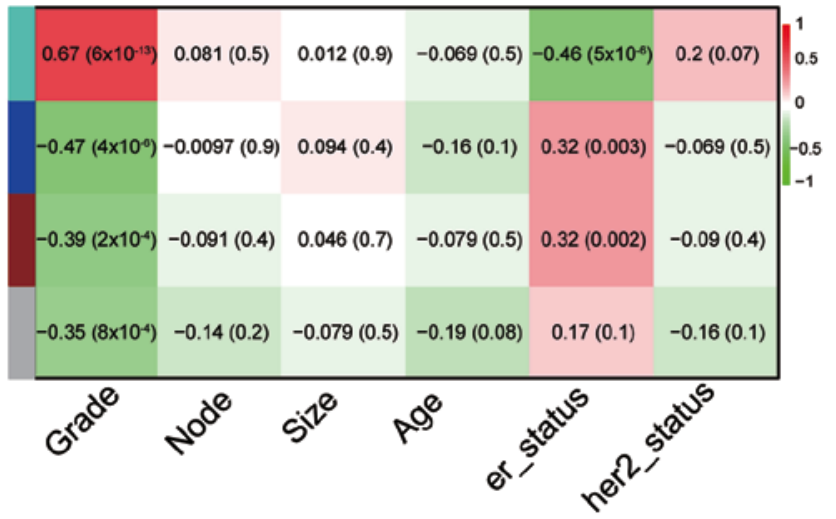

Module

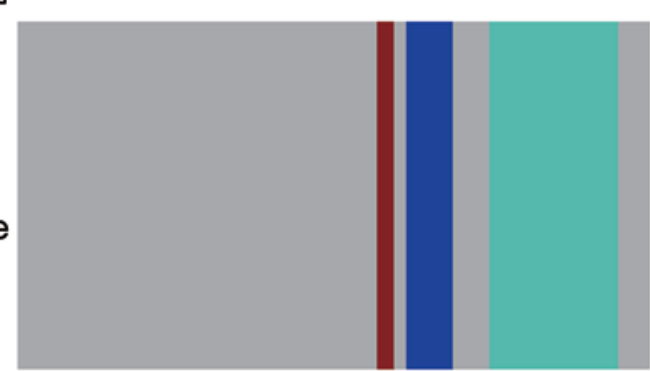

Figure 1. Key modules identified in breast cancer. (A) Clustering dendrogram of the 88 breast cancer samples. (B) Gene clustering and module identification by weighted correlation network analysis of the GSE20711 dataset. (C) Heatmap of the correlations between modules and clinicopathological characteristics of breast cancer. ER, estrogen receptor; HER2, human epidermal growth factor receptor 2.

activity' and 'microtubule binding' (Fig. 2C). In addition, the KEGG pathway analysis revealed that the DEGs were mainly involved in 'cell cycle', 'oocyte meiosis', 'pyrimidine metabolism', 'p53 signaling pathway' and 'DNA 
A

A

0
0
0
0

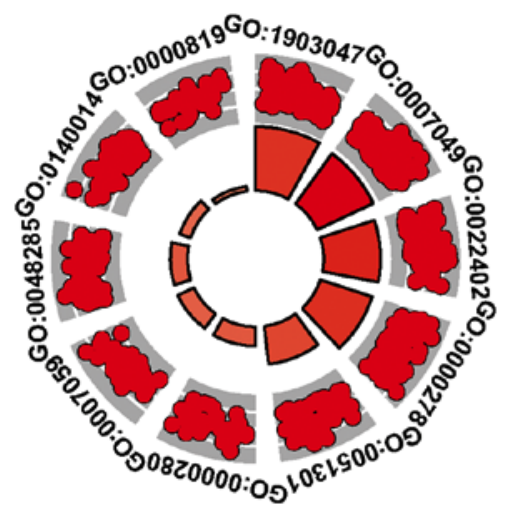

B

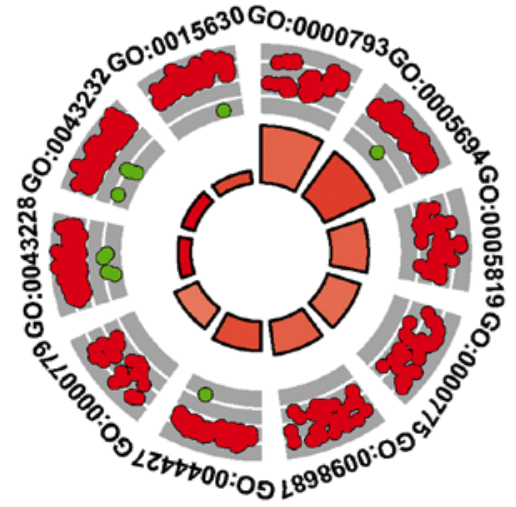

C

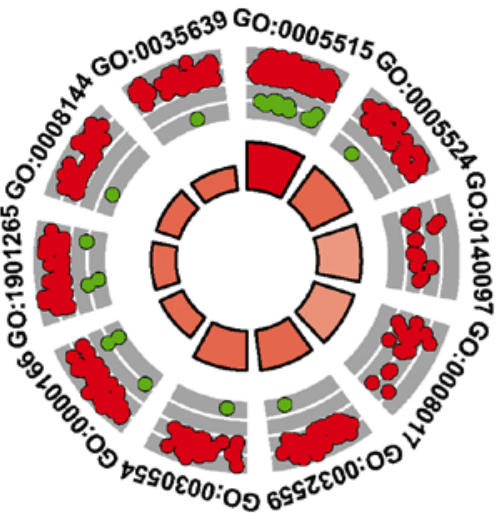

D

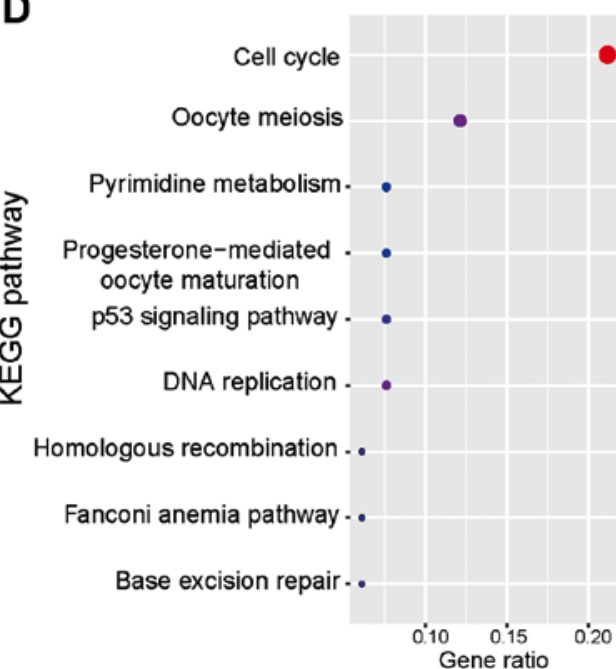

\begin{tabular}{|c|c|c|}
\hline ID & Description & z-score \\
\hline GO:1903047 & mitotic cell cycle process & Decreasing Increasing \\
\hline GO:0007049 & cell cycle & $\log \mathrm{FC}$ \\
\hline GO:0022402 & cell cycle process & - Downregulated \\
\hline GO:0000278 & mitotic cell cycle & Upregulated \\
\hline GO:0051301 & cell division & \\
\hline GO:0000280 & nuclear division & \\
\hline GO:0007059 & chromosome segregation & \\
\hline GO:0048285 & organelle fission & \\
\hline GO:0140014 & mitotic nuclear division & \\
\hline GO:0000819 & sister chromatid segregation & \\
\hline
\end{tabular}

\begin{tabular}{|c|c|}
\hline ID & $\begin{array}{c}\text { Description } \\
\text { GO:0000793 }\end{array}$ \\
\hline GO:0005694 & condensed chromosome \\
\hline GO:0005819 & chromosome \\
\hline GO:0000775 & spindle \\
\hline GO:0098687 & chromosome, centromeric region \\
\hline GO:0044427 & chromosomal part \\
\hline GO:0000779 & condensed chromosome, centromeric region \\
\hline GO:0043228 & non-membrane-bounded organelle \\
\hline GO:0043232 & intracellular non-membrane-bounded organelle \\
\hline GO:0015630 & microtubule cytoskeleton \\
\hline
\end{tabular}

\begin{tabular}{|c|c|}
\hline ID & Description \\
\hline GO:0005515 & protein binding \\
\hline GO:0005524 & ATP binding \\
\hline GO:0140097 & catalytic activity, acting on DNA \\
\hline GO:0008017 & microtubule binding \\
\hline GO:0032559 & adenyl ribonucleotide binding \\
\hline GO:0030554 & adenyl nucleotide binding \\
\hline GO:0000166 & nucleotide binding \\
\hline GO:1901265 & nucleoside phosphate binding \\
\hline GO:0008144 & drug binding \\
\hline GO:0035639 & purine ribonucleoside triphosphate binding \\
\hline
\end{tabular}
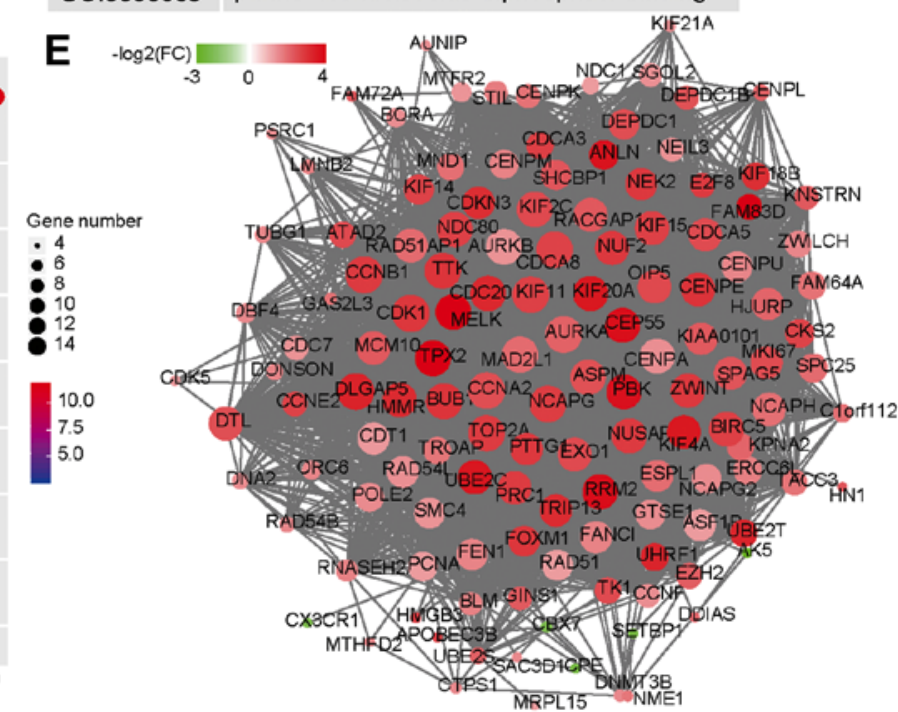

Figure 2. Enrichment analysis and PPI network of the genes in the turquoise module. (A) Results of the GO BP analysis, (B) the GO CC analysis and (C) the GO MF analysis of the turquoise module. The circles indicate the gene expression distribution in each term, and the Z-score value indicates the difference in the number of upregulated versus downregulated genes divided by the square root of the total count. (D) Results of the KEGG pathway analysis of the turquoise module. The colors indicate the significance [-log10(P-value)], and the size of the circles represents the number of genes enriched in the corresponding annotation. (E) The PPI network of the genes in the turquoise module. The size represents the degree of connectivity, and the color represents FC (red, upregulated genes; green, downregulated genes). GO, Gene Ontology; BP, biological process; CC, cellular component; MF, molecular function; PPI, protein-protein interaction; KEGG, Kyoto Encyclopedia of Genes and Genomes; FC, fold-change. 
Table I. Univariate Cox analysis and Kaplan-Meier survival analysis based on the data of patients with breast cancer from TCGA dataset.

\begin{tabular}{lccc}
\hline & \multicolumn{2}{c}{ Univariate analysis } & Kaplan-Meier survival analysis \\
\cline { 2 - 3 } Factor & HR $(95 \% \mathrm{CI})$ & P-value & Log-rank test P-value \\
\hline Pathological stage & $2.31(1.62-3.31)$ & $<0.001^{\mathrm{c}}$ & $<.001^{\mathrm{c}}$ \\
Lymph nodes & $2.31(1.6-3.33)$ & $0.019^{\mathrm{a}}$ & $>0.05$ \\
SHCBP1 & $1.18(1.03-1.35)$ & $0.034^{\mathrm{a}}$ & $0.006^{\mathrm{b}}$ \\
KIF21A & $1.19(1.01-1.39)$ & $0.027^{\mathrm{a}}$ & $0.010^{\mathrm{a}}$ \\
C80r76 & $1.27(1.03-1.58)$ & $0.035^{\mathrm{a}}$ & $0.008^{\mathrm{b}}$ \\
RAD54B & $1.2(1.01-1.41)$ & &
\end{tabular}

${ }^{\mathrm{a}} \mathrm{P}<0.05,{ }^{\mathrm{b}} \mathrm{P}<0.01$ and ${ }^{\mathrm{C}} \mathrm{P}<0.005$. HR, hazard ratio; $\mathrm{CI}$, confidence interval; RAD54B, RAD54 homolog B; KIF21A, kinesin family member 21A; C8orf76, chromosome 8 open reading frame 76; SHCBP1, SHC binding and spindle associated 1.

replication' (Fig. 2D). The PPI network based on the genes from the turquoise module demonstrated that the majority of the genes closely interacted with each other (Fig. 2E). Taken together, the results demonstrated that the DEGs in the clinically significant module mainly participated in the regulation of cancer cell proliferation, and that the majority of them interacted with each other.

Potential independent prognosis factors in breast cancer. The association of the turquoise module with tumor prognosis was analyzed based on TCGA data. Using univariate Cox analysis, several genes (SHCBP1, RAD54B, KIF21A and C8orf76) associated with prognosis were identified (Table I). The results of the Kaplan-Meier survival analysis identified RAD54B, KIF21A and C8orf76 as potential independent prognosis factors, which were negatively associated with overall patient survival time (Fig. 3A-C) and upregulated in late clinical tumor stages (III and IV) compared with early stages (I and II; Fig. S1).

The Human Protein Atlas database, the data of which are open access to all researchers, contains a systems-based analysis of protein expression in 17 types of cancer using data from 8,000 patients, as well as immunohistochemistry images directly demonstrating the indicated protein expression in tumor tissues $(37,38)$. The database was searched to analyze the expression of RAD54B, KIF21A and C8orf76 in normal and breast cancer tissues. With the exception of C8orf76, the protein expression of RAD54B and KIF21A was increased in breast cancer tissues compared with that in normal tissues (Fig. 3D-F).

RAD54B combined with TNM stage may predict the survival of patients with breast cancer. Since RAD54B, KIF21A and C8orf76 were significantly associated with the overall survival of patients with breast cancer, the patients from TCGA Project database were randomly divided into two groups: The discovery cohort and the internal cohort (Table SII). Combined with the clinicopathological characteristics in the datasets, a prognostic module was developed using forward conditional stepwise regression with multivariate Cox analysis in the discovery cohort. From the AIC values, a prognostic signature containing one gene (RAD54B) and one clinical trait (TNM stage) was identified. Based on this signature, the risk score was calculated for each sample in the discovery cohort using the following formula: Risk score=RAD54B expression $\times 0.236+\mathrm{TNM}$ stage $(\mathrm{I} / \mathrm{II}=0$ or $\mathrm{III} / \mathrm{IV}=1) \times 1.025$. The samples were ranked by score and divided into high-risk and low-risk groups based on the median of the risk scores (median, 1.915), which was set as the cut-off point (Fig. 4A). The sample with the median value was assigned to the high-risk group. The patients in the high-risk group exhibited shorter overall survival times compared with the patients in the low-risk group (Fig. 4B and C). In addition, the ROC curve analysis revealed that the AUC was 0.687 based on the risk scores (Fig. 4D).

The risk score model was further evaluated using the internal cohort. Using the risk-score formula and cut-off point derived from the discovery cohort, the patients were divided into high-risk $(n=256)$ and low-risk $(n=201)$ groups. The overall survival of the patients in the internal cohort revealed a similar trend to that in the discovery cohort (Fig. 4E-H). In addition, the nomogram based on the signature and the decision curve analysis demonstrated that this prognostic signature was effective for evaluating the prognosis of patients with breast cancer (Fig. S2).

Japonicone A may inhibit MCF-7 cell proliferation by targeting $R A D 54 B$. As RAD54B may serve an important role in breast cancer and may be a potential therapeutic target, the data from TCGA and GSE20711 were divided according to the expression of RAD54B into high and low expression groups (Fig. 5A and B). GSEA analysis demonstrated that the cell cycle function exhibited the strongest association with the expression of RAD54B in the two datasets (Fig. 5C and D). These results suggested that RAD54B may regulate the proliferation of breast cancer cells.

Based on the GSE85871 dataset, the expression of RAD54B in breast cancer MCF-7 cells treated with 102 different molecules used in traditional Chinese medicine was analyzed. The results demonstrated that treatment with a compound known as Japonicone A resulted in downregulated RAD54B expression in these cells (Fig. 6). To confirm this result, an inhibitory 


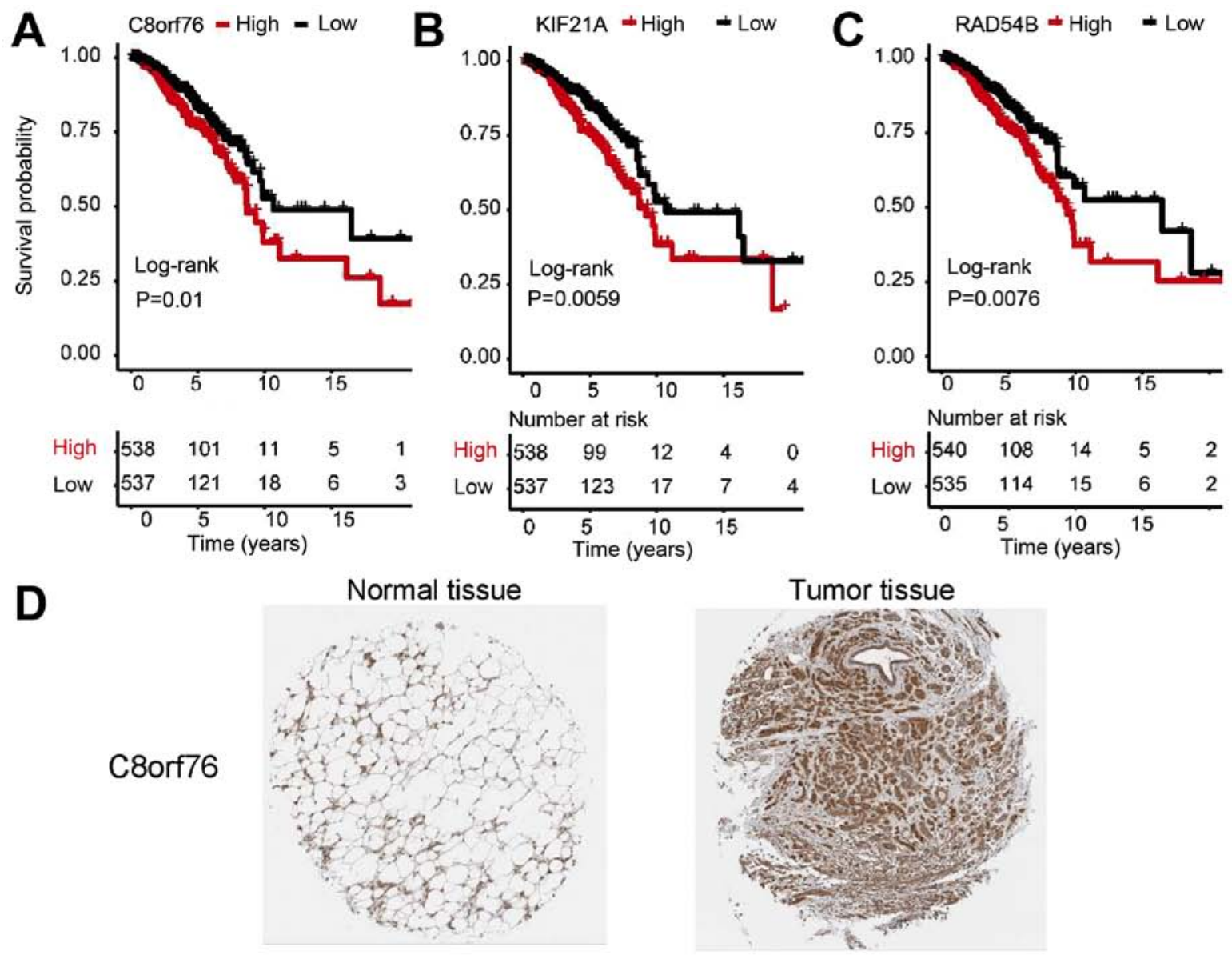

E

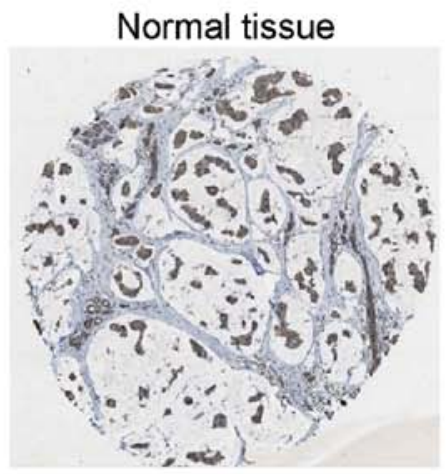

\section{Tumor tissue}

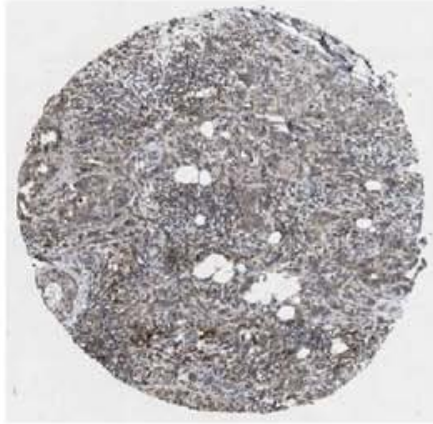

$\mathbf{F}$

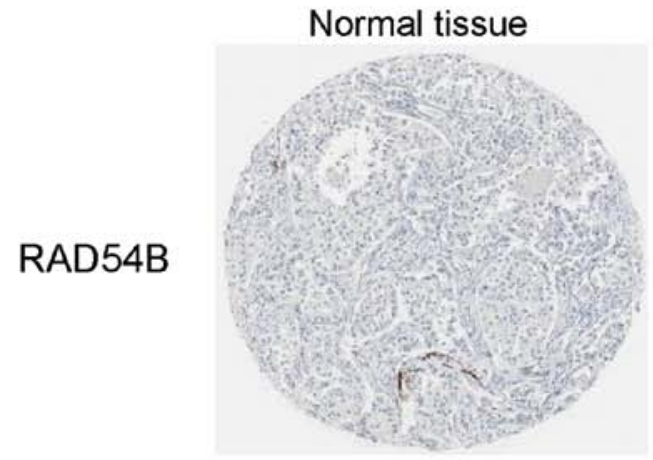

Tumor tissue

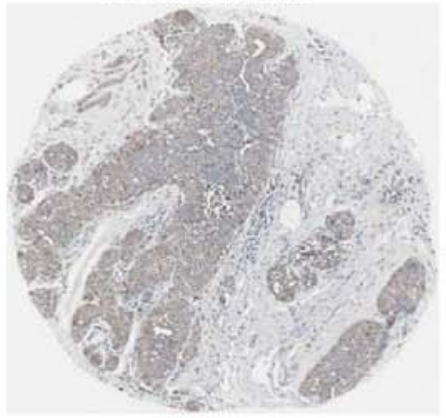

Figure 3. Overall survival and immunohistochemical analysis of the three genes identified in breast cancer. (A-C) Survival analysis based on Kaplan-Meier plotter; P-values were obtained from the log-rank test. Based on the median expression, the patients were classed into the high-level or the low-level group for (A) C8orf76, (B) KIF21A and (C) RAD54B. (D) The protein levels of C8orf76 in normal tissue (left: Staining, medium; intensity, moderate; quantity $>75 \%$ ) or tumor tissue (right: Staining, medium; intensity, moderate; quantity $>75 \%$ ). (E) The protein levels of KIF21A in normal tissue (left: Staining, low; intensity, weak; quantity $>75 \%$ ) or tumor tissue (right: Staining, medium; intensity, moderate; quantity $>75 \%$ ). (F) The protein levels of RAD54B in normal tissue (left: Staining, not detected; intensity, not detected; quantity, not detected) or tumor tissue (right: Staining, low; intensity, weak; quantity $>75 \%$ ). Images (D-F) were obtained from the Human Protein Atlas (http://www.proteinatlas.org/). RAD54B, RAD54 homolog B; KIF21A, kinesin family member 21A; C8orf76, chromosome 8 open reading frame 76. 
A
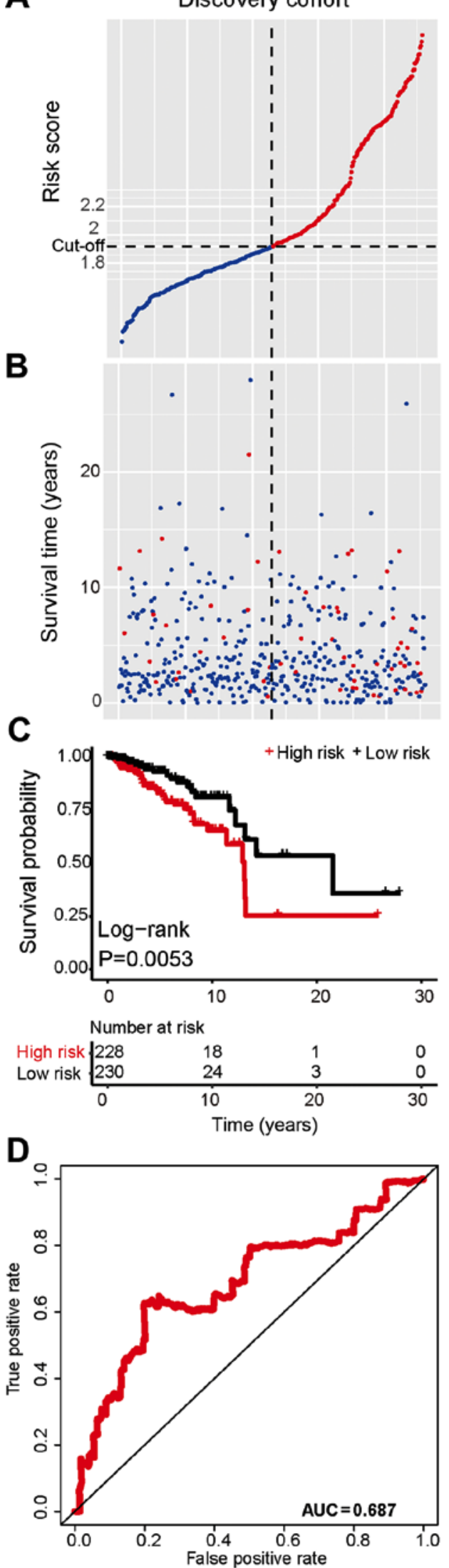

E

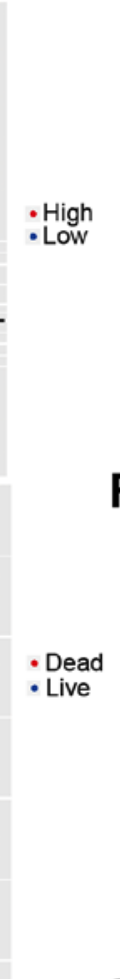

G

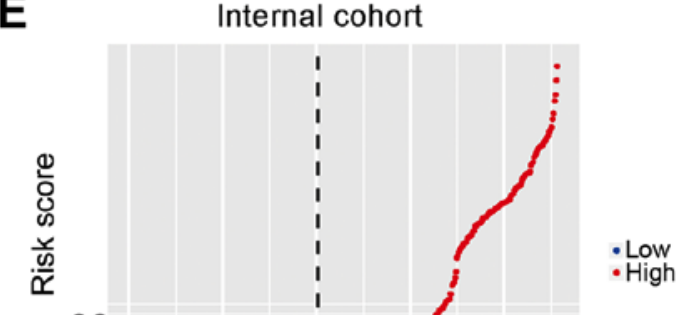

F

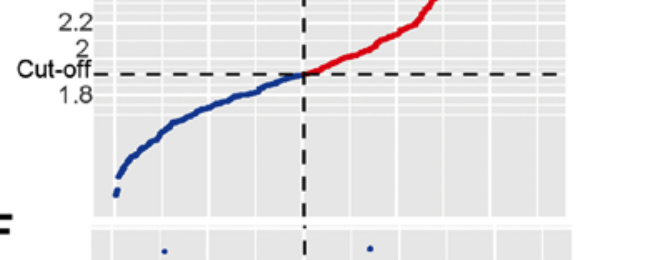

20.

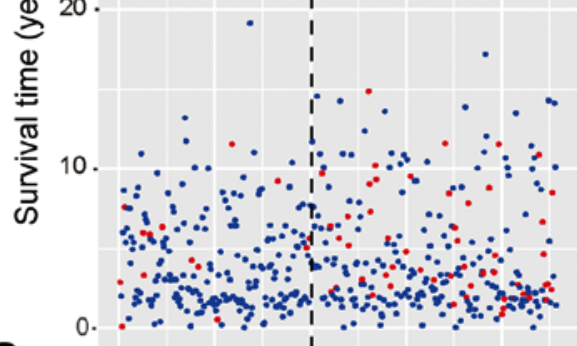

Dead

- Live

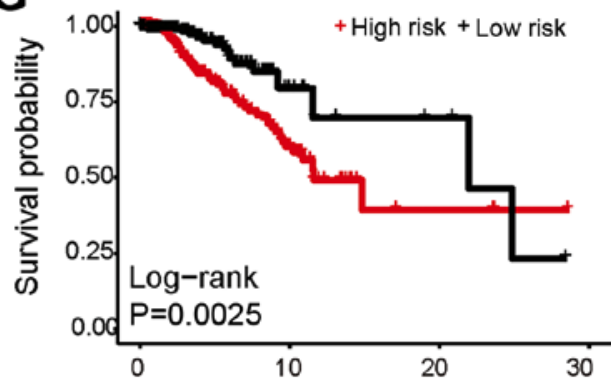

Number at risk

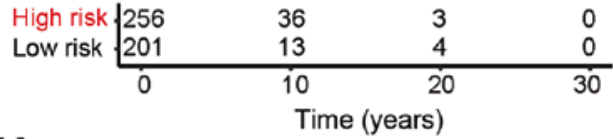

H

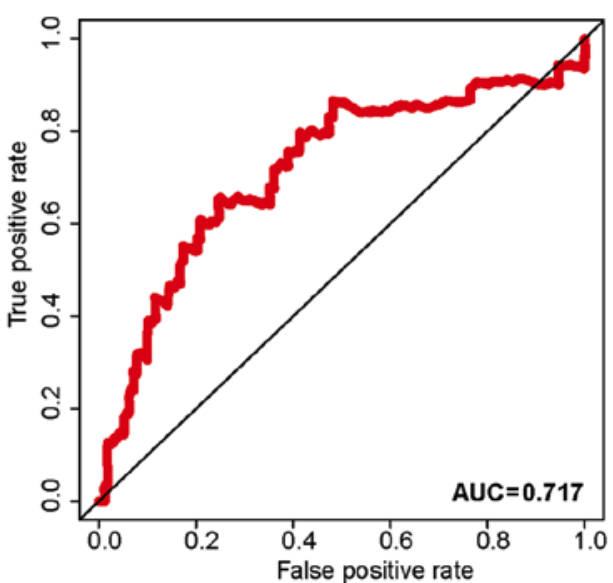

Figure 4. Signature (RAD54B expression and TNM stage) predictor score analysis of TCGA patients with breast cancer in the discovery and internal cohorts (A and E) The distribution of the risk score for the (A) discovery and (E) internal testing cohort. Each dot represents one patient; the vertical and horizontal axes represent the risk score calculated from the signature, and the results were sorted by risk score. The horizontal black dotted line represents the cut-off point. (B and F) Patient survival status and time in the (B) discovery and (F) internal testing cohort. Each dot represents one patient; the vertical axis represents the survival time. The results were sorted by the risk score. (C and G) Kaplan-Meier overall survival plots for TCGA breast cancer risk groups obtained from the (C) discovery and (G) internal testing cohort. (D and H) ROC curve analysis of the risk score in the (D) discovery (H) and internal testing cohort. TCGA, The Cancer Genome Atlas; RAD54B, RAD54 homolog B; ROC, receiver operating characteristic; AUC, area under the curve. 

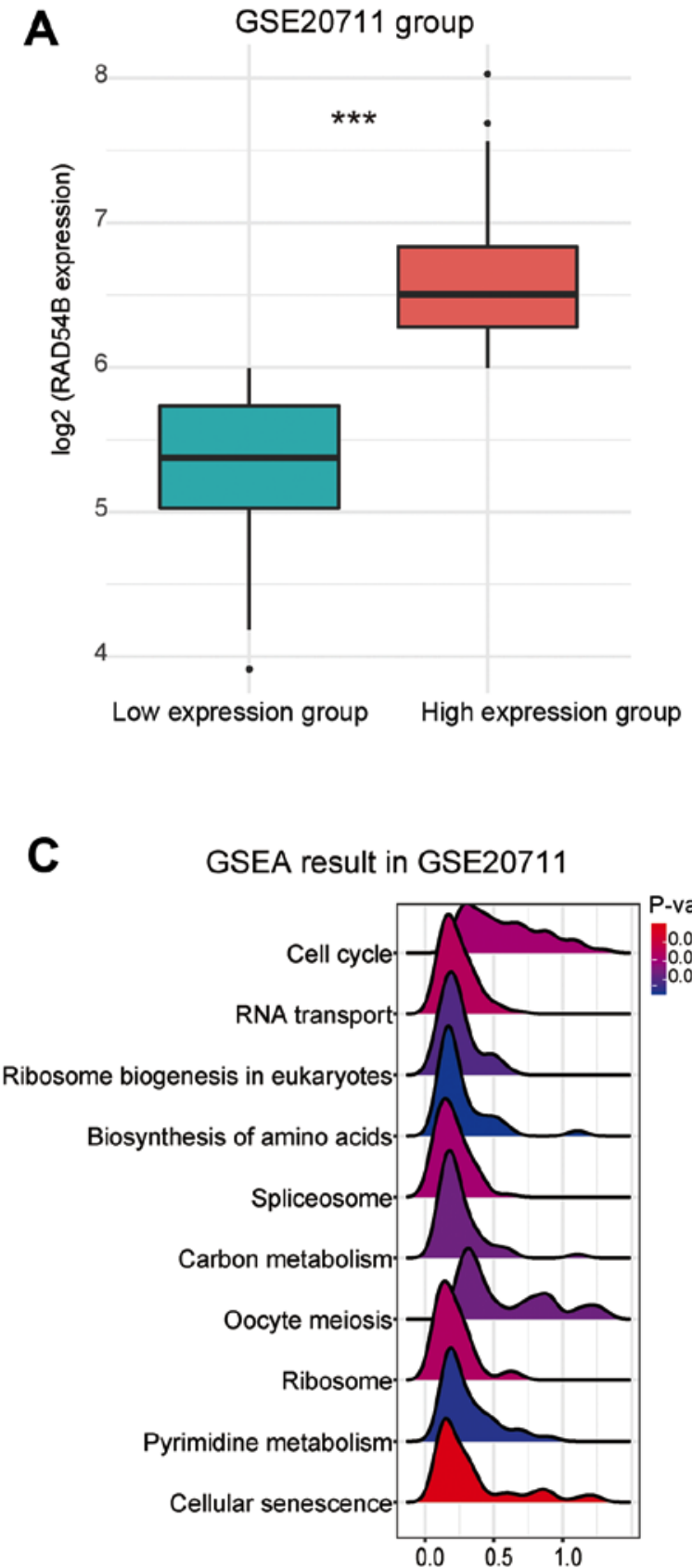

B

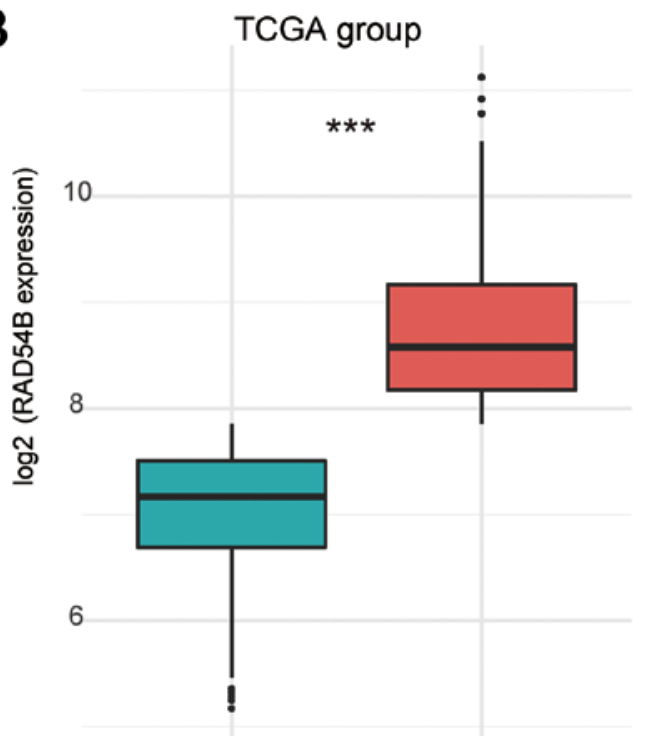

Low expression group High expression group
D

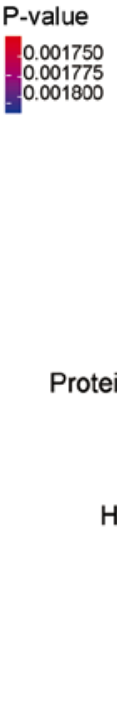

\section{GSEA result in TCGA}

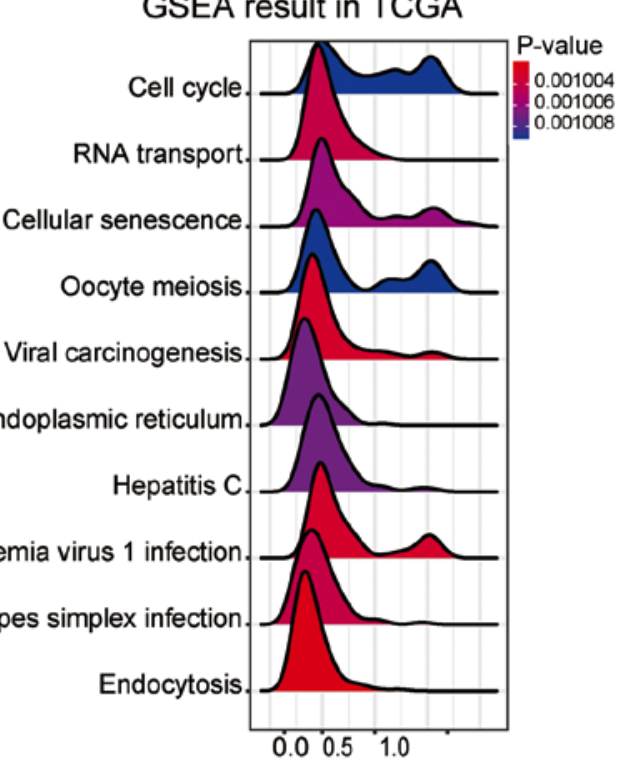

Figure 5. GSEA analysis of RAD54B high versus low expression groups in GSE20711 and TCGA. (A and B) The expression of RAD54B in the high and low expression groups in the (A) GSE20711 and (B) TCGA datasets. (C and D) Ridge plots of the results of the GSEA analysis of the (C) GSE20711 and (D) TCGA datasets. RAD54B, RAD54 homolog B; GSEA, Gene Set Enrichment Analysis; TCGA, The Cancer Genome Atlas. ${ }^{* * *} \mathrm{P}<0.005$ vs. low expression groups.

assay with Japonicone A on MCF-7 cells was performed. RT-qPCR analysis revealed that the expression of RAD54B was inhibited in MCF-7 cells treated with $10 \mu \mathrm{M}$ Japonicone A for $24 \mathrm{~h}$ (Fig. 7A). In addition, the proportion of $\mathrm{Ki}-67^{+} \mathrm{MCF}-7$ cells, which was the indicator for cell proliferation used in the present study, was reduced (Fig. 7B). The viability of MCF-7 cells was determined by MTT assay (39); the results demonstrated that cell viability was decreased following treatment with10 $\mu \mathrm{M}$ Japonicone A (Fig. 7C).

\section{Discussion}

Breast cancer is a major cause of mortality in females. Despite research on breast cancer treatment in the past decades, the currently available therapeutic strategies are still inadequate, especially for aggressive breast cancer (40). This is partly due to the lack of knowledge about the molecular pathogenesis of the disease. Therefore, exploring the molecular mechanisms and identifying biomarkers for breast cancer may provide more effective target-specific or personalized therapeutic strategies. In the current study, based on the data from the GSE20711 dataset, a sequential bioinformatics analysis was performed, and three genes that may have impact on the overall survival of patients with breast cancer were identified. One of the three genes, RAD54B, may be a useful prognostic factor for breast cancer.

RAD54B belongs to the Snf2 superfamily and maps to human chromosome 8q21.3-q22 (41). RAD54B was initially 


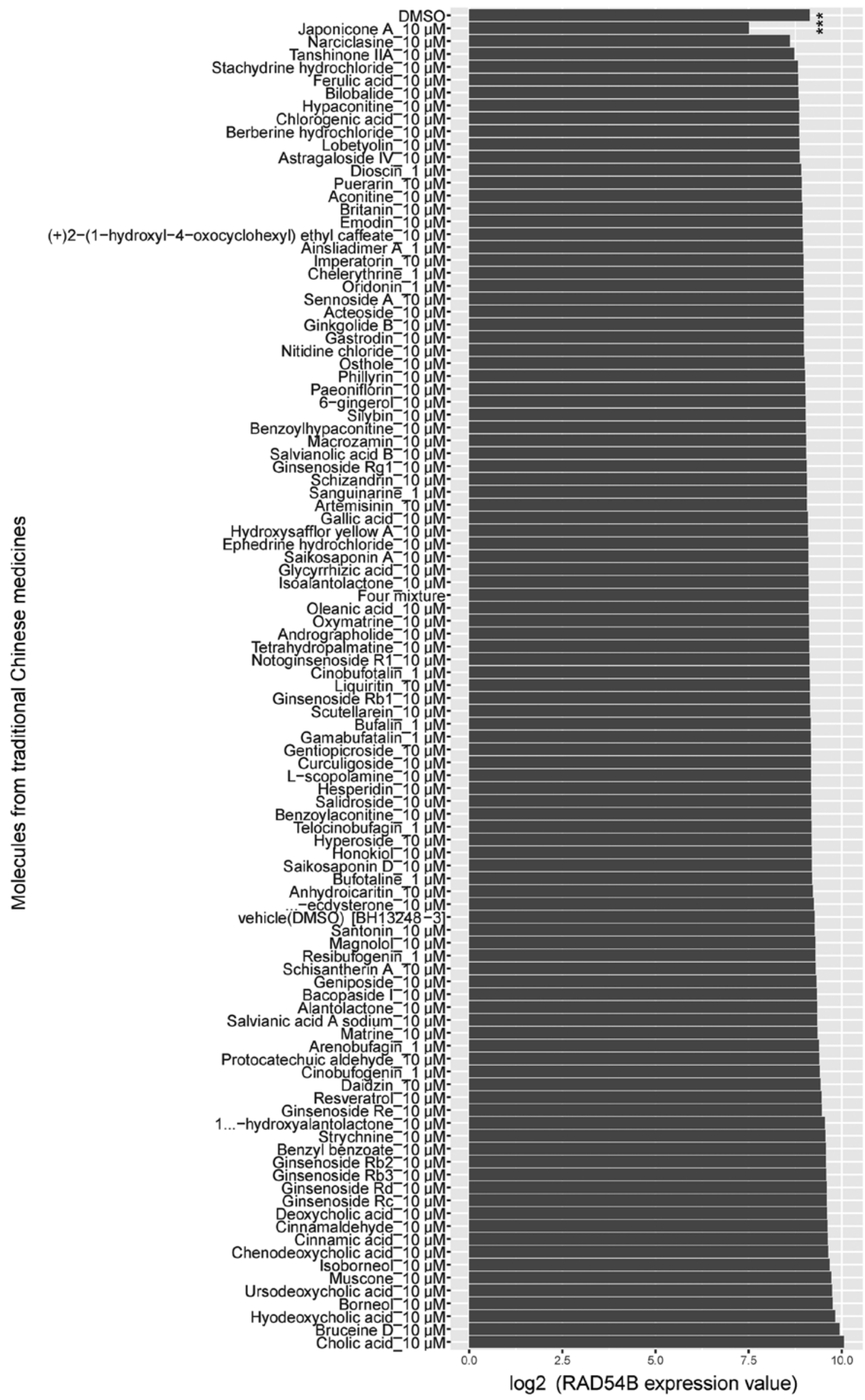

Figure 6. Expression of RAD54B in MCF-7 cells following treatment with 102 different molecules from traditional Chinese medicines, and only Japonicone A exhibited statistically significant inhibition on RAD54B. ${ }^{* * *} \mathrm{P}<0.001$ vs. DMSO. RAD54B, RAD54 homolog B. 
A

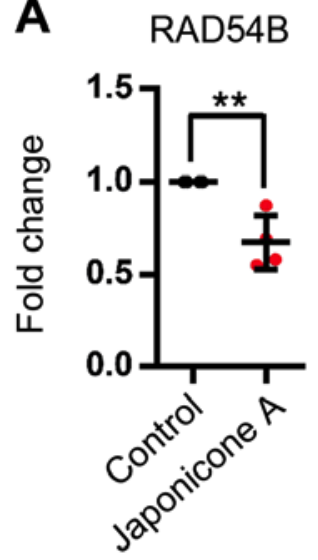

B

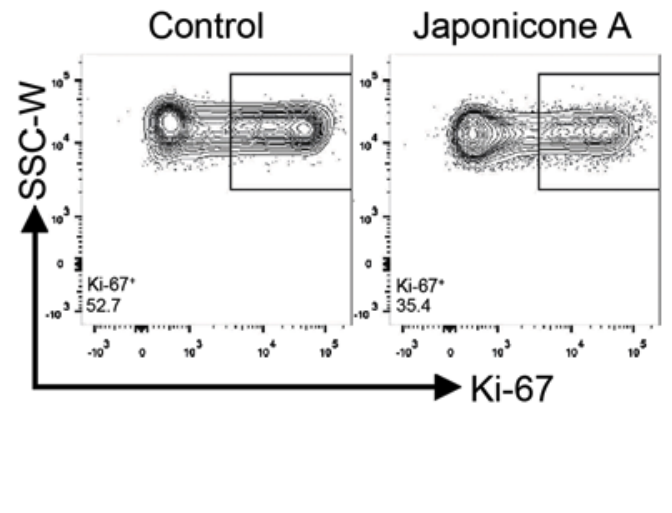

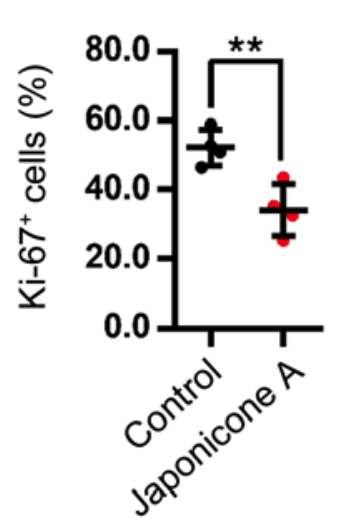

C

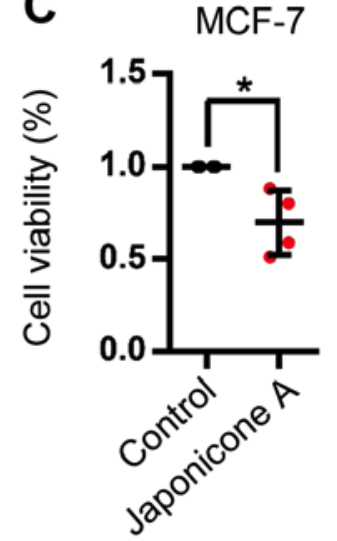

Figure 7. Effects of Japonicone A on the expression of RAD54B and the proliferation of MCF-7 cells. (A) The reverse transcription-quantitative PCR results of RAD54B expression in MCF-7 invasive ductal carcinoma cells following treatment with control (DMSO) or $10 \mu \mathrm{M}$ Japonicone A for $24 \mathrm{~h}$. (B) Flow cytometric analysis of the expression of ki-67 in MCF-7 cells following treatment with control or $10 \mu \mathrm{M}$ Japonicone A for $24 \mathrm{~h}$. SSC-W indicates the cellular granularity. (C) The MCF-7 cells were treated with control or $10 \mu \mathrm{M}$ Japonicone A for $24 \mathrm{~h}$, and an MTT assay was performed to assess the cell viability. All experiments were replicated four times and each dot in the histogram represents an independent experiment; error bars represent SD; horizontal lines represent the mean. ${ }^{*} \mathrm{P}<0.05,{ }^{* *} \mathrm{P}<0.01$. RAD54B, RAD54 homolog B; ki-67, marker of proliferation Ki-67.

identified as a homolog of RAD54, which serves a central role in homologous recombination and the DNA repair process (41-43). RAD54B also functions as a scaffold for p53 degradation in response to DNA damage, thus regulating the cell fate between cell cycle arrest and progression (37). As a result, constitutive upregulation of RAD54B promotes genomic instability; this has been observed in tumors, such as hepatic carcinoma (44). A recent study has demonstrated that inhibition of RAD54B significantly reduced the proliferation and colony formation of hepatoma cells (45). In the present study, RAD54B was upregulated in breast cancer cells, and its expression level was associated with the poor overall survival of patients with breast cancer. In addition, when combining RAD54B with patient TNM stage to construct a model, the model could be used to predict the prognosis of patients more accurately than TNM stage alone, which may be beneficial to the development of personalized treatments for patients. The results of the GO, KEGG and GSEA analyses demonstrated that RAD54B was primarily involved in the cell cycle regulation of breast cancer cells, and the in vitro inhibitory assay demonstrated that reduced expression of RAD54B significantly inhibited the proliferation of MCF-7 cells. Therefore, RAD54B may be used as a potential therapeutic target for breast cancer treatment.

Traditional Chinese medicine has been used for the prevention and treatment of diseases for centuries. Following the development of modern pharmacognosy, various biologically active natural compounds in traditional Chinese medicine, such as berberine and artemisinin, have been identified to exhibit therapeutic efficacy with minimal adverse effects, which provides new sources and platforms for developing first-line drugs (46-48). Thus, in combination with the aforementioned bioinformatics analysis, the data from the GSE85871 dataset, which includes the gene expression profiles of MCF-7 cells following treatment with 102 molecules from traditional Chinese medicine, were analyzed. The results demonstrated that Japonicone A effectively inhibited RAD54B expression in MCF-7 cells. Japonicone A is a component in the aerial parts of Inula japonica, which was traditionally used to treat bronchitis, digestive disorders, diabetes and general inflammation (49). Previous studies have demonstrated that Japonicone A may suppress the growth of Burkitt lymphoma cells via the NF- $\kappa$ B pathway (31) and the growth of non-small cell lung cancer cells via mitochondria-mediated pathways (50). In the present study, the bioinformatics analysis and the in vitro inhibitory assay revealed that Japonicone A may inhibit the expression of RAD54B in breast cancer cells, resulting in the inhibition of cell proliferation. In vivo experiments are required to provide stronger evidence; these will be performed in our future studies to explore the curative effect of Japonicone A on breast cancer.

In conclusion, the present study identified RAD54B as a prognostic factor and a potential therapeutic target for breast cancer.

\section{Acknowledgements}

Not applicable.

\section{Funding}

The current study was supported by the Special Research Fund of Chongqing Medical and Pharmaceutical College (grant no. ygz 2016103).

\section{Availability of data and materials}

The datasets analyzed during the current study are available in the Gene Expression Omnibus database (https://www.ncbi. nlm.nih.gov/geo). All other data generated or analyzed during this study are included in this published article.

\section{Authors' contributions}

$\mathrm{JF}$ and $\mathrm{JH}$ performed the experiments and analyzed the data. YX conceived and designed the experiments, supervised the study and wrote the manuscript. All authors read and approved the final manuscript. 


\section{Ethics approval and consent to participate}

Not applicable.

\section{Patient consent for publication}

Not applicable.

\section{Competing interests}

The authors declare that they have no competing interests.

\section{References}

1. Bray F, Ferlay J, Soerjomataram I, Siegel RL, Torre LA and Jemal A: Global cancer statistics 2018: GLOBOCAN estimates of incidence and mortality worldwide for 36 cancers in 185 countries. CA Cancer J Clin 68: 394-424, 2018.

2. Harbeck N and Gnant M: Breast cancer. Lancet 389: 1134-1150, 2017.

3. Redig AJ and McAllister SS: Breast cancer as a systemic disease: A view of metastasis. J Intern Med 274: 113-126, 2013.

4. Stratton MR, Campbell PJ and Futreal PA: The cancer genome. Nature 458: 719-724, 2009.

5. Balmain A, Gray J and Ponder B: The genetics and genomics of cancer. Nat Genet 33 (Suppl): S238-S244, 2003.

6. Nguyen DX and Massague J: Genetic determinants of cancer metastasis. Nat Rev Genet 8: 341-352, 2007.

7. Irish JM, Kotecha N and Nolan GP: Mapping normal and cancer cell signalling networks: Towards single-cell proteomics. Nat Rev Cancer 6: 146-155, 2006.

8. Papaleo E, Gromova I and Gromov P: Gaining insights into cancer biology through exploration of the cancer secretome using proteomic and bioinformatic tools. Expert Rev Proteomics 14 1021-1035, 2017

9. Aftab A, Shahzad S, Hussain HMJ, Khan R, Irum S and Tabassum S: CDKN2A/P16INK4A variants association with breast cancer and their in-silico analysis. Breast Cancer 26 : 11-28, 2019.

10. Klahan S, Wong HS, Tu SH, Chou WH, Zhang YF, Ho TF Liu CY, Yih SY, Lu HF, Chen SC, et al: Identification of genes and pathways related to lymphovascular invasion in breast cancer patients: A bioinformatics analysis of gene expression profiles Tumour Biol 39: 1010428317705573, 2017.

11. Tang J, Kong D, Cui Q, Wang K, Zhang D, Gong Y and Wu G: Prognostic genes of breast cancer identified by gene co-expression network analysis. Front Oncol 8: 374, 2018.

12. Cheng D, He $\mathrm{H}$ and Liang B: A three-microRNA signature predicts clinical outcome in breast cancer patients. Eur Rev Med Pharmacol Sci 22: 6386-6395, 2018.

13. Dedeurwaerder S, Desmedt C, Calonne E, Singhal SK, Haibe-Kains B, Defrance M, Michiels S, Volkmar M, Deplus R, Luciani J, et al: DNA methylation profiling reveals a predominant immune component in breast cancers. EMBO Mol Med 3 . 726-741, 2011.

14. Lv C, Wu X, Wang X, Su J, Zeng H, Zhao J, Lin S, Liu R, Li H, $\mathrm{Li} \mathrm{X}$ and Zhang $\mathrm{W}$ : The gene expression profiles in response to 102 traditional Chinese medicine (TCM) components: A general template for research on TCMs. Sci Rep 7: 352, 2017.

15. Mounir M, Lucchetta M, Silva TC, Olsen C, Bontempi G Chen X, Noushmehr H, Colaprico A and Papaleo E: New functionalities in the TCGAbiolinks package for the study and integration of cancer data from GDC and GTEx. PLoS Comput Biol 15:e1006701, 2019.

16. Ritchie ME, Phipson B, Wu D, Hu Y, Law CW, Shi W and Smyth GK: limma powers differential expression analyses for RNA-sequencing and microarray studies. Nucleic Acids Res 43: e47, 2015 .

17. Wen Q, Yang Y, Chen XH, Pan XD, Han Q, Wang D, Dang Y, $\mathrm{Li}$ XH, Yan J and Zhou JH: Competing endogenous RNA screening based on long noncoding RNA-messenger RNA co-expression profile in Hepatitis B virus-associated hepatocarcinogenesis. J Tradit Chin Med 37: 510-521, 2017.

18. Langfelder $P$ and Horvath S: WGCNA: An R package for weighted correlation network analysis. BMC Bioinformatics 9: 559, 2008.
19. Yuan L, Zeng G, Chen L, Wang G, Wang X, Cao X, Lu M, Liu X, Qian G, Xiao Y and Wang X: Identification of key genes and pathways in human clear cell renal cell carcinoma (ccRCC) by co-expression analysis. Int J Biol Sci 14: 266-279, 2018.

20. Wang T, Wu B, Zhang X, Zhang M, Zhang S, Huang W, Liu T, $\mathrm{Yu} \mathrm{W}, \mathrm{Li} \mathrm{J}$ and $\mathrm{Yu} \mathrm{X}$ : Identification of gene coexpression modules, hub genes, and pathways related to spinal cord injury using integrated bioinformatics methods. J Cell Biochem: Jan 17 , 2019 (Epub ahead of print). doi: 10.1002/jcb.27908.

21. Yu G, Wang LG, Han Y and He QY: ClusterProfiler: An R package for comparing biological themes among gene clusters. OMICS 16: 284-287, 2012.

22. Walter W, Sánchez-Cabo F and Ricote M: GOplot: An R package for visually combining expression data with functional analysis. Bioinformatics 31: 2912-2914, 2015.

23. Mo XG, Liu W, Yang Y, Imani S, Lu S, Dan G, Nie X, Yan J, Zhan R, Li X, et al: NCF2, MYO1F, S1PR4, and FCN1 as potential noninvasive diagnostic biomarkers in patients with obstructive coronary artery: A weighted gene co-expression network analysis. J Cell Biochem: Jan 17, 2019 (Epub ahead of print).

24. Yang Y, Lu Q, Shao X, Mo B, Nie X, Liu W, Chen X, Tang Y, Deng $Y$ and Yan J: Development of a three-gene prognostic signature for hepatitis B virus associated hepatocellular carcinoma based on integrated transcriptomic analysis. J Cancer 9: 1989-2002, 2018

25. Wang N, Guo H, Dong Z, Chen Q, Zhang X, Shen W, Bao Y and Wang X: Establishment and validation of a 7-microRNA prognostic signature for non-small cell lung cancer. Cancer Manag Res 10: 3463-3471, 2018.

26. Colwill K; Renewable Protein Binder Working Group and Gräslund S: A roadmap to generate renewable protein binders to the human proteome. Nat Methods 8: 551-558, 2011.

27. Xu H, Zhang Y, Qi L, Ding L, Jiang H and Yu H: NFIX Circular RNA promotes glioma progression by regulating miR-34a-5p via notch signaling pathway. Front Mol Neurosci 11: 225, 2018.

28. Subramanian A, Tamayo P, Mootha VK, Mukherjee S, Ebert BL, Gillette MA, Paulovich A, Pomeroy SL, Golub TR, Lander ES and Mesirov JP: Gene set enrichment analysis: A knowledge-based approach for interpreting genome-wide expression profiles. Proc Natl Acad Sci USA 102: 15545-15550, 2005.

29. Hu Z, Qin J, Zhang H, Wang D, Hua Y, Ding J, Shan L, Jin H, Zhang $\mathbf{J}$ and Zhang W: Japonicone A antagonizes the activity of TNF- $\alpha$ by directly targeting this cytokine and selectively disrupting its interaction with TNF receptor-1. Biochem Pharmacol 84: 1482-1491, 2012.

30. Qin JJ, Jin HZ, Fu JJ, Hu XJ, Wang Y, Yan SK and Zhang WD: Japonicones A-D, bioactive dimeric sesquiterpenes from Inula japonica Thunb. Bioorg Med Chem Lett 19: 710-713, 2009.

31. Li X, Yang X, Liu Y, Gong N, Yao W, Chen P, Qin J, Jin H, Li J, Chu R, et al: Japonicone A suppresses growth of Burkitt lymphoma cells through its effect on NF-кB. Clin Cancer Res 19: 2917-2928, 2013.

32. Du Y, Gong J, Tian X, Yan X, Guo T, Huang M, Zhang B, $\mathrm{Hu} \mathrm{X}$, Liu H, Wang Y, et al: Japonicone A inhibits the growth of non-small cell lung cancer cells via mitochondria-mediated pathways. Tumour Biol 36: 7473-7482, 2015.

33. Livak KJ and Schmittgen TD: Analysis of relative gene expression data using real-time quantitative PCR and the 2(-Delta Delta C(T)) method. Methods 25: 402-408, 2001

34. Patrick J: survivalROC: Time-dependent ROC curve estimation from censored survival data, https://cran.r-project.org/web/packages/survivalROC/index.html. Accessed May, 2019.

35. Vickers AJ and Elkin EB: Decision curve analysis: A novel method for evaluating prediction models. Med Decis Making 26: 565-574, 2006

36. Marshall B: rmda: Risk model decision analysis, https:// cran.r-project.org/web/packages/rmda/index.html. Accessed March 20,2018.

37. Uhlen M, Zhang C, Lee S, Sjöstedt E, Fagerberg L, Bidkhori G, Benfeitas R, Arif M, Liu Z, Edfors F, et al: A pathology atlas of the human cancer transcriptome. Science 357: pii eaan2507, 2017.

38. Pontén F, Jirström K and Uhlen M: The human protein atlas-a tool for pathology. J Pathol 216: 387-393, 2008

39. Zhou J,Li G, Zheng Y, Shen HM, Hu X, Ming QL, Huang C, Li P and Gao N: A novel autophagy/mitophagy inhibitor liensinine sensitizes breast cancer cells to chemotherapy through DNM1L-mediated mitochondrial fission. Autophagy 11: 1259-1279, 2015.

40. Nagini S: Breast cancer: Current molecular therapeutic targets and new players. Anticancer Agents Med Chem 17: 152-163, 2017. 
41. Hiramoto T, Nakanishi T, Sumiyoshi T, Fukuda T, Matsuura S, Tauchi H, Komatsu K, Shibasaki Y, Inui H ,Watatani M, et al: Mutations of a novel human RAD54 homologue, RAD54B, in primary cancer. Oncogene 18: 3422-3426, 1999.

42. Miyagawa K, Tsuruga T, Kinomura A, Usui K, Katsura M, Tashiro S, Mishima H and Tanaka K: A role for RAD54B in homologous recombination in human cells. EMBO J 21: 175-180, 2002.

43. Wesoly J, Agarwal S, Sigurdsson S, Bussen W, Van Komen S, Qin J, van Steeg $\mathrm{H}$, van Benthem J, Wassenaar E, Baarends WM, et al: Differential contributions of mammalian Rad54 paralogs to recombination, DNA damage repair, and meiosis. Mol Cell Biol 26: 976-989, 2006.

44. Yasuhara T, Suzuki T, Katsura M and Miyagawa K: Rad54B serves as a scaffold in the DNA damage response that limits checkpoint strength. Nat Commun 5: 5426, 2014.

45. Wang R, Li Y, Chen Y, Wang L, Wu Q, Guo Y, Li Y, Liu J and Wang L: Inhibition of RAD54B suppresses proliferation and promotes apoptosis in hepatoma cells. Oncol Rep 40: 1233-1242, 2018.
46. Mathur S and Hoskins C: Drug development: Lessons from nature. Biomed Rep 6: 612-614, 2017.

47. Clardy J and Walsh C: Lessons from natural molecules. Nature 432: 829-837, 2004.

48. Ertl P and Schuffenhauer A: Cheminformatics analysis of natural products: Lessons from nature inspiring the design of new drugs. Prog Drug Res 66: 217, 219-235, 2008.

49. Qin JJ, Jin HZ, Zhu JX, Fu JJ, Hu XJ, Liu XH, Zhu Y, Yan SK and Zhang WD: Japonicones E-L, dimeric sesquiterpene lactones from Inula japonica Thunb. Planta Med 76: 278-283, 2010.

50. West AP, Khoury-Hanold W, Staron M, Tal MC, Pineda CM, Lang SM, Bestwick M, Duguay BA, Raimundo N, MacDuff DA, et al: Mitochondrial DNA stress primes the antiviral innate immune response. Nature 520: 553-557, 2015.

(i) () () This work is licensed under a Creative Commons Attribution-NonCommercial-NoDerivatives 4.0 International (CC BY-NC-ND 4.0) License. 